\title{
Multiple Notification Modalities and Older Users
}

\author{
David Warnock \\ warnockd@dcs.gla.ac.uk
}

\author{
Marilyn McGee-Lennon \\ marilyn.mcgee- \\ lennon@glasgow.ac.uk
}

\author{
Stephen Brewster \\ stephen.brewster@glasgow.ac.uk
}

\author{
Glasgow Interactive Systems Group, School of Computing Science \\ University of Glasgow, Glasgow, G12 8QQ, UK
}

\begin{abstract}
Multimodal interaction can make home care reminder systems more accessible to their users, most of whom are older and/or have sensory impairments. Existing research into the properties of different notification modalities have used younger participants rather than members of the older population at which they are aimed. This paper presents the results of a user study with older adults that examined how different notification modalities affected $(a)$ performance in a card matching game and $(b)$ how effective the different modalities were at delivering information. Participants were all aged over 50 and notifications were delivered using textual, pictographic, abstract visual, speech, Earcon, Auditory Icon, tactile and olfactory modalities while playing the game. The results showed that older users were influenced by the same factors as younger users, yet there were subjective differences. The implications for the design of multimodal reminder systems for home care are discussed.
\end{abstract}

\section{Author Keywords \\ Multimodal; Older Users; Notifications; Reminders}

\section{ACM Classification Keywords}

H.5.2 [Information Systems Applications]: interaction styles

\section{INTRODUCTION}

The increasing availability of multimodal technology has created a world where messages can be delivered in a variety of ways through various sensory modalities. This is of benefit to people with sensory impairments, as developers now have the power to create more accessible technology which can use alternative modalities to communicate via non-impaired channels. This is particularly important for home care and reminder technology, where the primary users are elderly and often have one or more sensory impairments [1].

Many researchers have called for systems that offer multiple modalities and are able to switch between them to suit the user, environment or message being delivered [2, 6, 7, 9, 12]. Research has shown that adaptable technology such as this can deliver notifications in a manner that is more acceptable

Permission to make digital or hard copies of all or part of this work for personal or classroom use is granted without fee provided that copies are not made or distributed for profit or commercial advantage and that copies bear this notice and the full citation on the first page. To copy otherwise, or republish, to post on servers or to redistribute to lists, requires prior specific permission and/or a fee.

CHI 2013, April 27-May 2, 2013, Paris, France.

Copyright 2013 ACM 978-1-4503-1899-0/13/04...\$15.00. to its users $[4,6,8]$, while at the same time making such technology more robust and appropriate. Creating such a system however relies on a strong understanding of the target users and the comparative performance of the different modalities.

Warnock et al. [9, 10] argued that "there is no "best modality'; there is a best modality for the situation", and that the most appropriate modality should be selected based on a number of factors such as message urgency, user impairment and social context. Vastenburg et al. [8] found that delivering low/high urgency information using less/more salient methods increased user acceptability. Arroyo \& Selker's CarCoach [4] demonstrated that controlling when and how notifications were delivered lead to increased compliance and decreased frustration.

Arroyo \& Selker $[3,2]$ compared the performance of various modalities and found that notification modality did not affect performance in a primary task. This was confirmed by Warnock et al. [10] who evaluated text, pictogram, abstract visual, speech, Earcon, Auditory Icon, tactile and olfactory modalities. Warnock et al. [9] also found that target notifications (which required a response) and distractor notifications (which should be ignored) would induce the same negative effects (reduced success rate and pause in activity) regardless of the delivery modality. That work was carried out with younger users aged 18-30, but was extended to include older participants aged 50-90 for a direct comparison of modal notification performance between age groups [11]. They found that negative effects of notification delivery were much more pronounced for older participants. However, that work did not examine the differences between target and distractor notifications with older persons. As research suggests that older users have different expectations and requirements from technology than younger users $[7,12]$, there is a need to further investigate multimodal reminder design for this group.

This paper presents an experiment to investigate the disruptiveness and performance of different notification modalities when delivering target and distractor notifications to older users. This information could be used to create more effective notifications by outlining the relative performance, negative effects and appropriate applications of various notification modalities. This work is needed to inform the development of home care and reminder systems.

\section{EXPERIMENT DESIGN \& METHOD}

An experiment was conducted to evaluate the disruptiveness of notifications in different modalities which replicated the experiment described by Warnock et al. [9]. The experiment 
used a within-subjects design consisting of a primary task (a simple card matching game) and a range of unimodal notifications which instructed the participant to carry out a brief secondary task (pressing a button). The notifications used were identical to those used by Warnock et al. [9] to allow a direct comparison with earlier work.

The independent variables were:

- The modality of the notification (text, pictogram, abstract visual, speech, Earcon, Auditory Icon, tactile and olfactory modalities)

- The notification function (target vs. distractor)

The dependent variables were:

- The error rate in the primary task

- The longest pause post-notification

- Notification reaction time

- Notification response accuracy

The experiment was carried out with 15 participants (6 male, 9 female), all between the ages of 50 and 90 .

\section{Primary Task}

The task chosen was a simple card-matching game called 'Concentration' (also known as Memory or Pairs) as used by Warnock et al. $[9,11]$. In Concentration, pairs of cards are presented face-down to the player. The player can then turn over two cards per turn in an attempt to find the matching pairs and remove them from the game. Concentration is a simple leisure activity that might well be carried out at home, it is a well-known game with simple rules and it can quickly build a mental workload. The game was configured as it was by Warnock et al. [9, 10]; the cards showed simple alphabet caricatures, with each game using 24 cards and a 60 second time limit.

\section{Secondary Task}

The secondary task was to press a single, large yellow button when instructed to do so by a target notification. This button was placed directly in front of the participant within easy reach as shown in Figure 1a. There were two different notification functions in the experiment: target notifications required the participant to press the yellow button, while distractor notifications were to be completely ignored.

\section{Notification Design}

The notifications used in the experiment were identical to those used by Warnock et al. [9]: for each of the 8 modalities there were 3 different notifications representing either 'heating', 'lights' or 'telephone'. Text and pictogram notifications were delivered directly into the game window to the top of the play area. The abstract visual display used a short-throw projector to display a coloured light against a wall, deliberately aligned to appear in the peripheral vision of the participant as shown in Figure 1b. In all audio conditions (speech, Earcons and Auditory Icons) notifications were delivered through headphones. Tactile notifications were delivered via an EAI C2 vibrotactile actuator (www.eaiinfo.com) secured to the top of the wrist on the non-dominant hand with

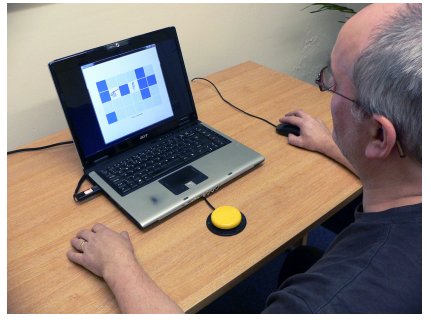

(a) Experimental Setup

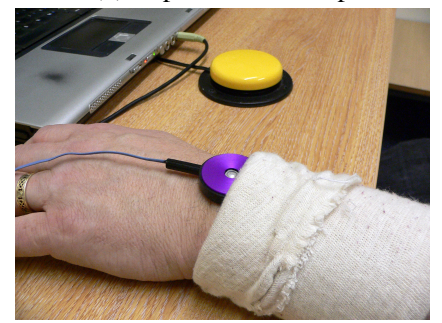

(c) Tactile Device

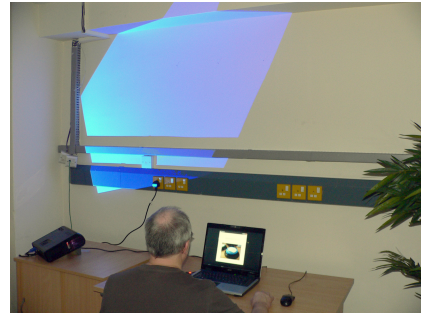

(b) Abstract Visual

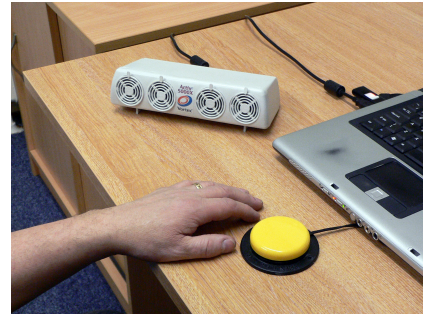

(d) Olfactory Device
Figure 1: Hardware used in the experiment.

a stretchable bandage as shown in Figure 1c. Olfactory notifications were delivered using a Dale Air (www.daleair.com) Vortex Active smell device as shown in Figure 1d, which delivered the scents of chocolate, eucalyptus and raspberries. These were stored on fabric disks which were blown by a fan to deliver the smell. Full specifications and justification can be found in [9].

\section{Measurements}

Warnock et al. $[9,11]$ developed a number of measures relating to the game concentration. Error rate was measured as superfluous views per click, where a card was repeatedly viewed without matching it to another. Considering the timing of notifications, Warnock et al. identified that notification delivery would create a pause in game activity; the length of this pause, in seconds, was called the longest pause and represents the time taken to process a notification. This paper presents an additional metric; reaction time, the time in seconds from the initiation of delivery to the start of the longest pause. Notification response accuracy considers if the notifications were correctly acknowledged or ignored.

\section{Hypotheses}

The aim of the experiment was to evaluate if the conclusions made by Warnock et al. [9, 10] hold true for older participants. The first hypothesis considers the impact of the notifications on the primary task, as follows:

H1: Neither notification function nor modality will have an effect on the error rate in the primary task (superfluous views per click) and there will not be an interaction effect between notification function and modality.

The second set of hypotheses considers the performance of the notifications themselves, which was found by Warnock $e t$ al. to be heavily affected by modality: 


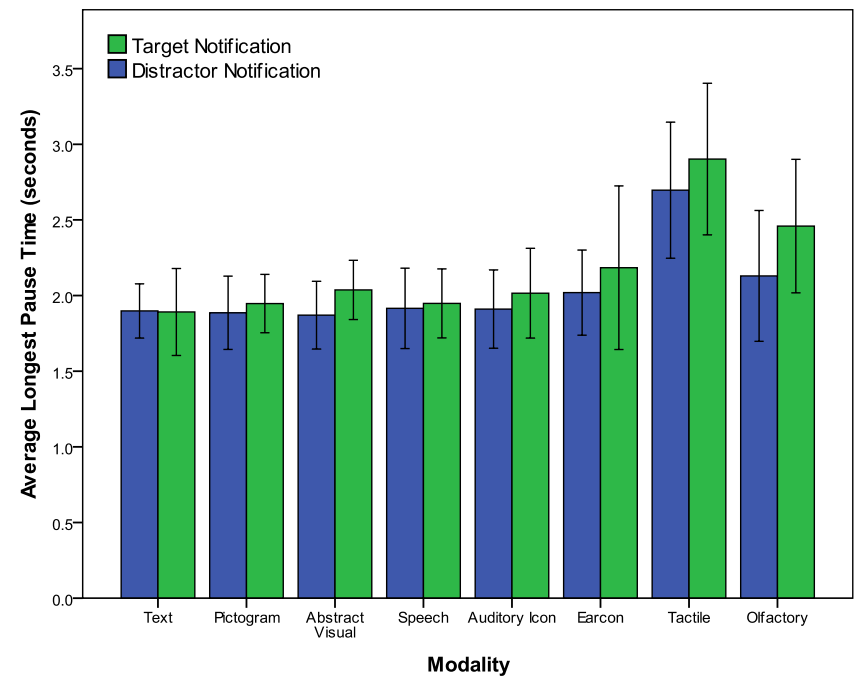

Figure 2: Graph showing the average pause time for target and distractor notifications in different modalities. Error bars show $95 \%$ confidence intervals.

H2: Notification modality and notification function will have an effect on the pause time post-notification (seconds) but there will be no interaction effect between the factors.

H3: Modality will affect reaction time (seconds);

H4: Notification function will not affect reaction time (seconds).

\section{Procedure}

At the start of the experiment, participants practised playing the game and ignoring/acknowledging simple practice notifications. The main experiment consisted of 8 experimental conditions (one for each modality) and a control condition (with no notifications) with the conditions delivered in a random order. Each experimental condition consisted of a training segment and 4 games. During the training the participant was exposed to all 3 notifications before the target was randomly selected. The training would test the participant's ability to recognise the target and differentiate it from the distractors. In each condition 6 target and 6 distractor notifications were randomly delivered, 3 per game, over 4 games. At the end of the experiment a short interview was carried out focussing on which modalities participants liked/disliked and how they would feel about having them at home. The experiment took around 75 minutes.

\section{RESULTS}

\section{Primary Task Performance}

As with younger users, no relationship was found between modality and error rate in the primary task. The target and distractor notifications did not produce a significant effect on error rate and no interaction effects were found between the two factors, confirming Hypothesis 1.

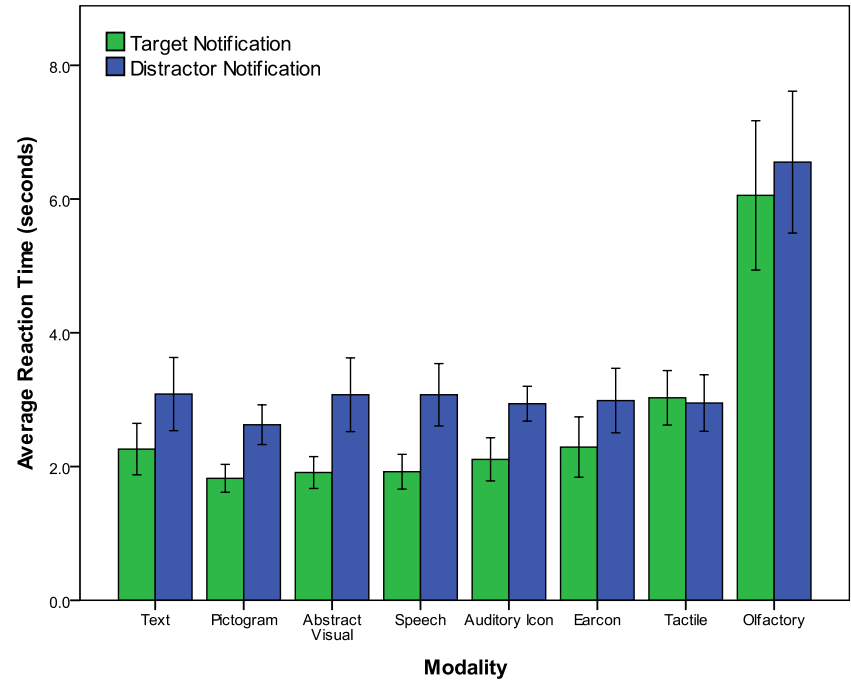

Figure 3: Graph showing the average response times for target and distractor notifications in different modalities. Error bars show $95 \%$ confidence intervals.

\section{Secondary Task Performance}

When considering the longest pause (the theoretical time taken to process a notification), a 2-factor ANOVA found a significant main effect of both modality $(F(7,98)=11.53$, $\left.p<0.001, \eta_{p}^{2}=.45\right)$ and notification function $(F(1,14)=$ 8.93, $\left.p<0.05, \eta_{p}^{2}=.39\right)$. No interaction effect was found. The evidence supports Hypothesis 2 and is shown in Figure 2. Despite notification function having an effect on the pause time, the real-world difference is very low. This will be explored further in the discussion.

Reaction time was measured as the time from the initiation of delivery to the start of the longest pause. When examining if modality affected reaction time, the test failed Mauchly's test of sphericity $\left(\chi^{2}(27)=84.6, p<0.01\right)$, so the result was corrected with the Greenhouse-Geisser correction $(\epsilon=0.28)$. Analysis showed modality had a significant main effect on reaction time $(F(1.98,27.74)=54.7, p<0.001$, $\eta_{p}^{2}=.77$ ), supporting Hypothesis 3. Notification function was also found to have a significant main effect on reaction time $\left(F(1,14)=89.55, p<0.001, \eta_{p}^{2}=.87\right)$, which does not support Hypothesis 4, as shown in Figure 3. In addition to this, an interaction effect was found between modality and notification type on reaction speed $(F(7,98)=2.9$, $\left.p<0.05, \eta_{p}^{2}=.14\right)$.

\section{DISCUSSION}

The findings of this experiment confirm that the findings of Warnock et al. [9] are also valid for older people. Firstly, no significant differences were found in error rate after the delivery of target and distractor notifications. This suggests that distractor notifications have a similar negative impact on error rate as target notifications.

With younger participants, Warnock et al. [9] reported that the distractor notifications create a longer pause than target 
notifications did, although the real-world difference was very small. That result suggested that younger participants were able to quickly resolve notifications for the target information and return to the game. As shown in Figure 2, in this case it is the distractor notifications which were resolved quickest. This makes sense as the participants had to mentally process the notification then press the yellow button shown in Figure 1a. The real-world difference between the longest pause times is very small at 0.15 seconds, again highlighting that the cost of a distraction is surprisingly high.

One interesting new finding is that participants seem to become aware of certain target notifications more quickly than similar (i.e. the same modality and style) distractor notifications, as shown by the shorter reaction time visible in Figure 3 . The interaction with modality is interesting; there was a very large difference for abstract visual, but no real difference for tactile. It may be that participants were somehow subconsciously 'looking' for important information, and switched attention faster when it was detected. The results also show that this process did not allow participants to completely ignore distractors, resulting in attention being drawn to both notifications.

It is possible that participants were unable to ignore distractors because of their similarity to the targets. It may be easier to ignore distractors in different modalities, although further tests would be needed to confirm this. Experience could also play a role here; participants only had a small amount of time to practise differentiating between targets and distractors. The difference between modalities could also be explained by the properties of the sensory channel in use; for example, people are generally good at picking out important information in their peripheral vision, but they are not necessarily good at processing tactile or smell information.

It is important to consider subjective input from participants; if the technology is unpopular, it will not be welcomed into user's homes. In post-test interviews, older participants reacted very differently than younger participants to the different modalities. Despite younger and older participants exhibiting similar performance in the olfactory condition, younger participants were much more positive about smell while almost all of the older participants expressed negative sentiments. Participants were generally positive about the other modalities. Many participants stated that the abstract visual method was highly salient, although participants expressed different opinions on whether this was a good thing or not. Unlike with younger participants, older participants were highly aware of how these methods could be employed to interact with people who have sensory impairments.

\section{FUTURE WORK \& CONCLUSIONS}

The results of this experiment contribute further to our understanding of how we interact in different modalities. This field has grown with the work of Warnock et al. [9, 10, 11], Vastenburg et al. [8], and Arroyo \& Selker [3, 2]. Future work should consider how these notification modalities could be used to create better home care and reminder systems for older users and users with significant sensory impairments. Future work should also consider using combinations of modalities, which have been shown to be more effective than unimodal notifications for older people [5].

In conclusion, older users will be affected by the same factors as younger ones when receiving notifications in different modalities. Care must be taken to avoid the negative effects of delivering notifications in the home, such as an increased risk of mistakes in other tasks. The results presented here contribute to a growing body of knowledge that gives developers a better understanding of the performance and abilities of different notification modalities. These findings can be used to create more accessible, adaptable, and appropriate multimodal technology for the home.

\section{ACKNOWLEDGEMENTS}

We would like to thank our experimental participants and the EPSRC for funding this work (grant EP/G069387/1).

\section{REFERENCES}

1. National Service Framework for Older People. UK Department of Health (2007).

2. Arroyo, E. and Selker, T. Self-Adaptive Multimodal-Interruption Interfaces. In International Conference on Intelligent User Interfaces (2003), 6-11.

3. Arroyo, E., Selker, T. and Stouffs, A. Interruptions as Multimodal Outputs: Which are the Less Disruptive? In Proceedings. Fourth IEEE International Conference on Multimodal Interfaces, IEEE Comput. Soc (2002), 479-482.

4. Arroyo, E., Sullivan, S. and Selker, T. CarCoach: A Polite and Effective Driving Coach. In CHI 'O6 extended abstracts on Human factors in computing systems - CHI '06, CHI EA '06, ACM Press (Montreal, Canada, 2006), 357-362.

5. Emery, V. K., Edwards, P. J., Jacko, J. A., Moloney, K. P., Barnard, L., Kongnakorn, T., Sainfort, F. and Scott, I. U. Toward Achieving Universal Usability for Older Adults Through Multimodal Feedback. Proceedings of the 2003 conference on Universal usability - CUU'O3 (2003), 46.

6. McBryan, T., McGee-Lennon, M. and Gray, P. An Integrated Approach to Supporting Interaction Evolution in Home Care Systems. PETRA 282 (2008), 45:1-45:8.

7. McGee-Lennon, M., Wolters, M. and Brewster, S. User-Centred Multimodal Reminders for Assistive Living. In CHI ' 11 (Vancouver, CA, 2011), 2105-2114.

8. Vastenburg, M. H., Keyson, D. V. and de Ridder, H. Considerate Home Notification Systems: A User Study of Acceptability of Notifications in a Living-Room Laboratory. International Journal of Human-Computer Studies 67, 9 (Sept. 2009), 814-826.

9. Warnock, D., McGee-Lennon, M. and Brewster, S. The Impact of Unwanted Multimodal Notifications. In Proceedings of the 13th international conference on multimodal interfaces - ICMI'11, ICMI '11, ACM Press (Alicante, Spain, 2011), 177-184.

10. Warnock, D., McGee-Lennon, M. and Brewster, S. The Role of Modality in Notification Performance. In INTERACT 2011, M. Campos, Pedro and Graham, Nicholas and Jorge, Joaquim and Nunes, Nuno and Palanque, Philippe and Winckler, Ed., vol. 6947 of Lecture Notes in Computer Science, Springer Berlin / Heidelberg (Portugal, Spain, 2011), 572-588.

11. Warnock, D., McGee-Lennon, M. and Brewster, S. Older Users, Multimodal Reminders and Assisted Living Technology. Journal of Health Informatics 18, 3 (2012), 181-190.

12. Xiao, B., Lunsford, R., Coulston, R., Wesson, M. and Oviatt, S. Modeling Multimodal Integration Patterns and Performance in Seniors Toward Adaptive Processing of Individual Differences. In Proceedings of the 5th international conference on Multimodal interfaces, ACM (2003), 265-272. 